\title{
Subsidy design: wealth versus benefits
}

\author{
Simona Grassi · Ching-to Albert Ma
}

Received: 21 May 2009 / Accepted: 4 May 2010 / Published online: 22 May 2010

(C) Springer-Verlag 2010

\begin{abstract}
A government would like to subsidize an indivisible good. Consumers' valuations of the good vary according to their wealth and benefits from the good. A subsidy scheme may be based on consumers' wealth or benefit information. We translate a wealth-based policy to a benefit-based policy, and vice versa, and give a necessary and sufficient condition for the pair of policies to implement the same assignment: consumers choose to purchase the good under the wealth-based policy if and only if they choose to do so under the translated benefit-based policy. General taxation allows equivalent policies to require the same budget.
\end{abstract}

Keywords Subsidy design - Wealth-based policies - Benefit-based policies . Assignment set - Translation between subsidy policies · Equivalence between subsidy policies $\cdot$ Cost-effectiveness $\cdot$ Means-testing

JEL Classification H2 - Taxation, Subsidies, and Revenue - H42 - Publicly Provided Private Goods · H5 - National Government Expenditures and Related Policies · I38 - Government Policy; Provision and Effects of Welfare Programs

\section{Introduction}

Governments and public organizations often subsidize goods and services such as education and health care. Subsidies are often based on partial information. In Canada and

\footnotetext{
S. Grassi (ه)

Institute of Health Economics and Management - IEMS, Faculty of Business and Economics, Université de Lausanne, Route de Chavannes 31, 1015 Lausanne, Switzerland e-mail: simona.grassi@unil.ch

C. A. Ma

Department of Economics, Boston University, 270 Bay State Road, Boston, MA 02215, USA e-mail: ma@bu.edu
} 
many European countries, subsidies under national health services are based on illness severity or expected treatment benefit, but not wealth. Such subsidies implement a form of cost-effectiveness. In the United States, the Medicaid and state programs provide subsidized health insurance for low-income individuals or families, and typically do not ration services according to illness. Such subsidies implement a form of meanstesting. Similarly, in European public universities subsidized fees are mostly based on family wealth, while in American private universities scholarships are mostly based on test scores, which can proxy the expected benefit from higher education. Usually, wealth-based subsidies help the poor while benefit-based subsidies help the more deserved.

In the context of an indivisible good, we show that wealth-based and benefitbased subsidies may share a common property. In the examples above, although cost-effectiveness and means testing apparently refer to different kinds of subsidies, we show that they are very much related. The concept we develop to connect between wealth-based and benefit-based policies is the assignment set, defined as the subset of all consumers who purchase the subsidized good. A wealth-based subsidy policy induces a set of consumers to purchase the good, so it implements an assignment set; likewise, for a benefit-based policy.

When will a pair of wealth-based and benefit-based policies implement the same assignment set? What are the properties of an assignment set implemented simultaneously by a pair of wealth-based and benefit-based policies? If an assignment set is implemented simultaneously, is the required budget under a wealth-based policy different from the equivalent benefit-based policy? What are the policy implications? We answer all these questions here.

We present a necessary and sufficient condition for wealth-based and benefit-based subsidy schemes to be assignment-equivalent, and an algorithm to translate between them. In the examples above, under our condition we can translate benefit-subsidies that implement cost-effectiveness to wealth-subsidies that implement means-testing, without changing the assignment set. In other words, cost-effectiveness may be implemented using wealth information instead of benefits information. Furthermore, the subsidies are decreasing in wealth, so wealthy consumers pay more.

Our analysis is relevant for information collection and processing, an important issue for policy implementation, but often dismissed in the literature. Under our necessary and sufficient condition, collecting either wealth or benefit information is sufficient for the implementation of an assignment set. If wealth information is unreliable due to tax evasion, the regulator can rely on benefit information alone. On the other hand, if a robust tax system has been in place so that wealth information is reliable and readily available, benefit information is unnecessary. Our result can guide regulator to save on information costs.

An assignment set represents one important dimension of the effect of a policy, even if an assignment set alone does not describe consumers' utilities. Assignmentequivalent wealth-based and benefit-based policies induce the same decision from consumers, but generally each consumer receives a different subsidy depending on the policy, and hence consumer utilities also differ. It does appear to us, however, that ensuring a set of consumers to obtain a good is an important and practical objective of many policies. 
Many public policies are framed in terms of assignment sets. For example, in 2002 President Bush signed the "No Child Left Behind" Act for primary education reform. ${ }^{1}$ A goal of the reform is to provide education to underprivileged children, and to ensure that all children achieve literacy by the third grade. Another example is illustrated by the recent UK higher education reform, aimed at increasing the number of new enrollments from low-income households. In his first statement to the House of Commons as Secretary of State for Innovation, Universities and Skills (July 2007), John Denham announced major increases in support to poorer students. In his words: "... everyone who has the potential and qualifications to succeed in higher education, whatever their family background, should have the opportunity to participate...We cannot be satisfied when only $28 \%$ of students come from low income backgrounds." 2

In both examples, politicians and policy makers determine the main goal, which may be interpreted as setting up an assignment set. In the "No Child Left Behind" example, the assignment set consists of children with limited family resources. In the UK higher education reform example, the assignment set consists of young people with suitable academic abilities and limited family wealth.

Financing issues such as required budgets, tax burdens, and actual subsidies to students are important, but are decentralized to local authorities. In the UK higher education reform, the Department of Innovation, Universities and Skill seeks to implement the assignment set through an income-based subsidy policy. In our framework, this corresponds to a wealth-based subsidy policy. The use of a wealth-based subsidy policy to foster participation of poorer students in higher education seems natural. However, financial aids can also be based on academic achievements, and they are quite common. Academic achievement may be a proxy for students' expected benefit from higher education. ${ }^{3}$ As in the health care examples, we show that the assignment set implemented by a wealth-based policy may be implemented by a benefit-based policy.

Our results yield new insights and interpretations. We show that for wealth-based and benefit-based schemes that are equivalent, the subsidized price is increasing in wealth if and only if it is decreasing in benefit. A benefit-based policy encouraging consumption for the more deserved can only be assignment-equivalent to a wealth-based policy charging a higher price for the rich.

Generally, with limited tax and subsidy instruments, equivalent wealth-based and benefit-based schemes result in different revenues for the regulator. The budgets required under each scheme for the implementation of an assignment set are different. With more tax and subsidy instruments, such as lump sum or general taxes, equivalent schemes that require the same budget can be constructed.

\footnotetext{
1 See http://www.ed.gov/nclb/overview/intro/execsumm.html.

2 See http://www.berr.gov.uk/dius/science/page40318.html.

3 There is an extensive empirical literature on the correlation between abilities and returns to education. Academic achievement can be a proxy for ability. According to Patrinos et al. (2006), in low-income countries, abilities and returns seem to be negatively correlated while the opposite is true in high-income countries. In terms of a subsidy policy, when ability and returns are positively correlated, subsidizing students with higher grades may be optimal. Our setup is flexible enough to include both cases.
} 
Our approach departs from the conventional cost-benefit methodology. We neither assume a social welfare function nor focus on a fixed set of incentive issues. We introduce the concept of an assignment and explore properties of policies implementing it. It can be regarded as a "third-best" or satisficing approach. Our method, however, complements the usual "second-best" or optimizing approach for selecting among policies. Given an assignment, one can calculate consumers' cost burden and utilities under alternative equivalent subsidy schemes. This information may help a regulator to refine the choice among subsidy schemes.

The literature on the public provision of private goods is extensive. The main focus has been on the reasons for such provisions and the optimal design of tax and transfer schemes when consumers are heterogenous and possess private information. The missing information gives rise to the relevant incentive constraints. The equivalence between subsidy schemes based on different information has not been studied before.

Arrow (1971) gives a benchmark for the optimal public provision of private goods under perfect information. He lets individuals be different in abilities. For a utilitarian social welfare function he derives the optimal expenditure policy. The subsequent literature focuses on asymmetric information and incentive problems. Blackorby and Donaldson (1988) show that when the social planner cannot observe individuals' abilities or illness, in-kind transfers may be preferred over monetary transfers. Assuming that income information cannot be used by the social planner, Besley and Coate (1991) justify the public provision of private goods as a way to redistribute income from the rich to the poor.

Following Mirrlees (1971), Boadway and Marchand (1995) model the public provision of a private good in the context of optimal income taxation, where individuals have private information about their labor productivity. More recently, De Fraja (2002) investigates the design of optimal education policies, when children in households have different abilities, and when household incomes differ. In De Fraja's model, income is observable, but ability is private information.

Some of the literature on the optimal tax and subsidy design deals with inequality under asymmetric information. It is recognized that inequality depends on wealth, as well as characteristics such as age, health status, gender, etc. As a consequence, transfers should take into account these characteristics. Atkinson (1992) concludes that "the issue of policy design is not therefore a confrontation between fully universal benefits and pure income testing; rather the question is that of the appropriate balance of categorical and income tests." Blackorby and Donaldson (1994) study the optimal transfers between groups ("people with serious illness, the disabled, racial and ethnic groups," etc., p. 440) when the planner does not know the distribution of income within groups.

All of the above literature is concerned with the optimal design. Each paper focuses on a specific issue. The case of missing information about abilities and incomes has been a major focus while other issues such as distribution and inequality have also been studied. In line with the literature, we assume that the regulator must design a subsidy under partial information; either income or benefit information is available. Our investigation about the relationship between subsidy policies that are based on different information is new. 
We introduce the model in the next section. In Sect. 3, we first define an assignment set for a subsidy scheme. We then present the translation between wealth-based schemes and benefit-based schemes. A necessary and sufficient condition is presented for an assignment set to be implemented simultaneously by wealth-based and benefit-based policies. In Sect. 4, we first show that equivalent schemes generally collect different revenues, so require different budgets. Then we show that if the regulator can use more general tax or subsidy schemes, equivalent subsidy schemes can be constructed to collect the same revenue. In Sect. 5, we compare our approach with the conventional constrained optimization approach. We show that our necessary and sufficient condition for equivalent implementation of assignment sets may be consistent with socially optimal schemes. The last section draws some conclusions. An Appendix contains proofs and examples.

\section{The model}

A regulator allocates a private good to a set of consumers. The good is indivisible, but each unit of the good may give different benefits to different consumers. In both the education and health markets, there are many such examples. A course of study confers different benefits depending on students' abilities; a course of treatment or surgery may heal an illness, but consumers may experience different recovery utilities. Nevertheless, the cost of a study or treatment program may not vary according to consumer characteristics. ${ }^{4}$

We normalize the total mass of consumers to 1. Each consumer gets at most one unit of the good. Each unit of the good costs $c>0$. The regulator has available a budget $B$ to pay for these goods. We assume that $0 \leq B<c$, so the regulator cannot supply the good to all consumers for free. It is unimportant for the analysis whether the government produces the good or contracts with a firm to do so.

A consumer has wealth $w$. A consumer obtains benefit $\ell$ when he consumes the good. We let $w$ and $\ell$ be random variables. Respectively, the variables $w$ and $\ell$ have supports on positive and finite intervals $[\underline{w}, \bar{w}]$ and $[\underline{\ell}, \bar{\ell}]$. Let $F$ and $f$ be the distribution and density functions of $w$; let $G$ and $g$ be the distribution and density functions of $\ell$. We assume that these distributions are independent, but a correlation between $w$ and $\ell$ does not alter any of our results. Our theory is not about inferring the (conditional) distribution of $w$ given $\ell$, or vice versa. We say that a consumer is type $(w, \ell)$ if he has wealth $w$ and obtains benefit $\ell$ from the good.

If a type $(w, \ell)$ consumer pays $p$ to obtain the good, his utility is $U(w-p)+\ell$, where $U$ is a strictly increasing and strictly concave function. If a consumer does not obtain the good (and pays nothing), his utility is $U(w)$. The benefit $\ell$ is assumed to be separable from the utility of wealth; this assumption has no conceptual consequence for the analysis. ${ }^{5}$ Without loss of generality, the utility from benefit $\ell$ is measured

\footnotetext{
4 We do not consider cost selection issues here. For some services, the provision cost may well depend on consumer characteristics. See Grassi and Ma (2009).

5 In a more general setting, the utility functions are $U(w-p, \ell)$ and $U(w, 0)$. See also Footnote 8.
} 
linearly. ${ }^{6}$ In education for example, the variable $\ell$ measures his (expected) benefit from a course of study in utility units. The model works in a slightly different but isomorphic way in the health care setting. Here $\ell$ represents the (expected) loss of illness in utility units. If a sick consumer does not get treatment, his utility is $U(w)-\ell$ ; if he pays $p$ to obtain treatment his utility becomes $U(w-p)$.

A type $(w, \ell)$ consumer is willing to pay for the good at a price $p$ if

$$
U(w-p)+\ell \geq U(w) .
$$

The consumer's willingness to pay is monotone in wealth and benefit. If a type $(w, \ell)$ consumer is willing to pay for the good at price $p$, so are those who have higher wealth and those who derive higher benefits. For a type $\left(w^{\prime}, \ell^{\prime}\right)$ consumer, where $w^{\prime}>w$ and $\ell^{\prime}>\ell$, the following inequalities follow from (1):

$$
U\left(w^{\prime}-p\right)+\ell \geq U\left(w^{\prime}\right) \quad U(w-p)+\ell^{\prime} \geq U(w) .
$$

A wealth-based subsidy policy is a function $t:[\underline{w}, \bar{w}] \rightarrow \mathbb{R}^{+}$; a consumer pays $t(w)$ if he purchases the good. A benefit-based policy is a function $s:[\underline{\ell}, \bar{\ell}] \rightarrow \mathbb{R}^{+}$; a consumer pays $s(\ell)$ if he purchases the good. If the regulator intends to give subsidies, $s(\ell)$ and $t(w)$ will be less than $c$, the cost of the good, which is borne by the regulator or government. ${ }^{7}$

For most of the analysis, we let consumers pay nothing if they do not purchase the good from the regulator. In Sect. 4.1, we allow general subsidies. There, a wealthbased subsidy is a pair of functions $t_{1}:[\underline{w}, \bar{w}] \rightarrow \mathbb{R}$ and $t_{2}:[\underline{w}, \bar{w}] \rightarrow \mathbb{R}$, where $t_{1}(w)$ is the payment when the consumer does not buy the good and $t_{2}(w)$ is the payment when he does. Similarly, a benefit-based subsidy is a pair $s_{1}:[\underline{\ell}, \bar{\ell}] \rightarrow \mathbb{R}$ and $s_{2}:[\underline{\ell}, \bar{\ell}] \rightarrow \mathbb{R}$, where $s_{1}(\ell)$ is the payment when the consumer does not get the good and $s_{2}(\ell)$ is the payment when he does. These payments are allowed to be positive or negative. The payments that are imposed on consumers when they do not purchase the good may be regarded as general taxation or subsidy, or they may even be lump sum or poll taxes ( $t_{1}$ and $s_{1}$ being constant functions).

\section{Equivalent policies and assignments}

Consider a wealth-based policy $t:[\underline{w}, \bar{w}] \rightarrow \mathbb{R}^{+}$. We define the assignment set $\alpha(t)$ due to $t$ by

$$
\alpha(t) \equiv\{(w, \ell): U(w-t(w))+\ell \geq U(w)\}
$$

\footnotetext{
6 The utility from benefit $\ell$ can be written generally as $V(\ell)$, where $V$ is strictly increasing. We define a new benefit variable $\tilde{\ell} \equiv V(\ell)$ and adjust the distribution and density functions $G$ and $g$ accordingly.

7 If consumers have access to a private market, the regulator's policies will be constrained by the market price. For example, if a consumer can purchase the good in the private market at $d$ (which may be higher than $c$ ), then $s(\ell)$ and $t(w)$ must not be higher than $d$. The results are unaffected by this restriction.
} 
The inequality in (3) says that a type $(w, \ell)$ consumer prefers to buy the good at price $t(w)$. Next, consider a benefit-based policy $s:[\underline{\ell}, \bar{\ell}] \rightarrow \mathbb{R}^{+}$. We define the assignment set $\beta(s)$ due to $s$ by

$$
\beta(s) \equiv\{(w, \ell): U(w-s(\ell))+\ell \geq U(w)\}
$$

The inequality in (4) says that a type $(w, \ell)$ consumer prefers to buy the good at price $s(\ell)$. We consider subsidy policies that implement nonempty, proper assignment subsets of consumers.

When the wealth-based policy $t$ implements the assignment $\alpha(t)$, we can compute the required subsidy. The regulator is responsible for the balance $c-t(w)$. Hence the total subsidy under policy $t$ is

$$
\int_{\alpha(t)}[c-t(w)] \mathrm{d} F(w) \mathrm{d} G(\ell) .
$$

Similarly, when the benefit-based policy $s$ implements the assignment $\beta(s)$, the total subsidy is

$$
\int_{\beta(s)}[c-s(\ell)] \mathrm{d} F(w) \mathrm{d} G(\ell)
$$

\subsection{Translation between wealth-based and benefit-based policies}

We present the translation from a wealth-based subsidy policy to an equivalent benefit-based policy, and the translation in the opposite direction. We then discuss policy implications. Given $t$, and for any $w \in[\underline{w}, \bar{w}]$, define $\hat{\ell}$ by the equation:

$$
U(w-t(w))+\hat{\ell}=U(w) .
$$

A type $(w, \hat{\ell})$ consumer is indifferent between purchasing the good at $t(w)$ and the status quo. Equation (7) defines a functional relationship between $w$ and $\hat{\ell}$. We denote this function by $\theta:[\underline{w}, \bar{w}] \rightarrow \mathbb{R}$ and call $\theta$ the "indifference boundary" with respect to $t$ :

$$
\hat{\ell}=\theta(w ; t) \equiv U(w)-U(w-t(w))
$$

We suppress the policy $t$ in the argument of $\theta$. From (2), at each $w$, consumers with benefits $\ell>\hat{\ell}=\theta(w)$ strictly prefer to purchase the good.

The shape of the indifference boundary depends on the policy $t$. If $t$ is differentiable, the slope of the indifference boundary is

$$
\frac{\mathrm{d} \hat{\ell}}{\mathrm{d} w}=\left[U^{\prime}(w)-U^{\prime}(w-t(w))\right]+U^{\prime}(w-t(w)) t^{\prime}(w)
$$


Fig. 1 Increasing indifference boundary

Fig. 2 Decreasing indifference boundary

Fig. 3 Discontinuous indifference boundary
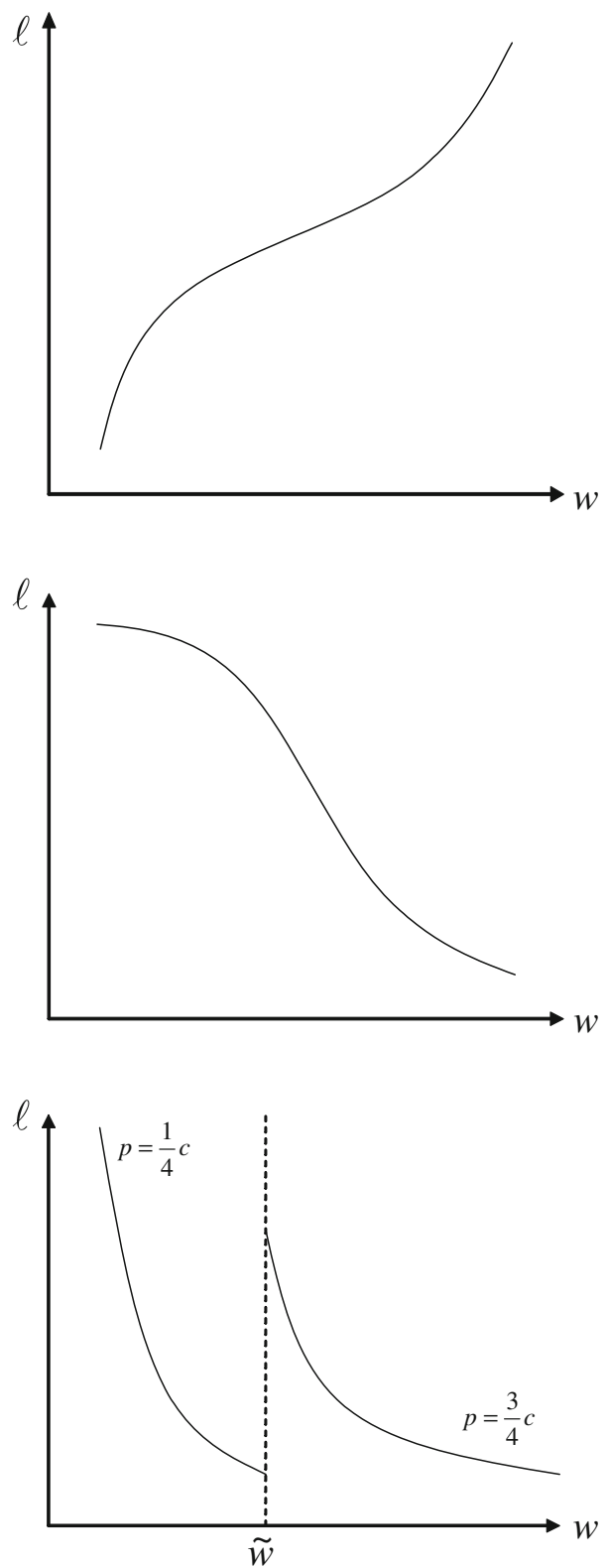

from the differentiation of (8). Because $U$ is strictly concave, the term inside the square brackets in (9) must be negative. If $t$ is decreasing $\left(t^{\prime}(w) \leq 0\right)$, or increasing but the value of $t^{\prime}(w)$ is not too large, the indifference boundary is negatively sloped.

Figures 1, 2, and 3 show three indifference boundaries. In Fig. 1, the boundary is increasing. Under this policy if a consumer with wealth $w$ and benefit $\ell$ is indifferent between paying $t(w)$ to obtain the good and not, a consumer with wealth $w^{\prime}>w$ 
actually declines to pay $t\left(w^{\prime}\right)$ to get the same benefit. The wealth-based policy is very progressive and increases so rapidly that the consumer must receive more benefit than $\ell$ to be willing to pay $t\left(w^{\prime}\right)$. In Fig. 2, the boundary is decreasing, and the comparison between the consumer's decisions at wealth levels $w$ and $w^{\prime}$ goes exactly the opposite way. In Fig. 3, the boundary is generated by a discontinuous policy: $t(w)=\frac{1}{4} c$ for $w<\widetilde{w}$ and $t(w)=\frac{3}{4} c$ otherwise.

Condition 1 (Decreasing indifference boundary) The wealth-based policy $t:[\underline{w}$, $\bar{w}] \rightarrow \mathbb{R}^{+}$is continuous (equivalently the function $\theta$ is continuous). The indifference boundary $\theta$ is strictly decreasing.

The indifference boundary in Fig. 2 satisfies Condition 1, while the one in Fig. 1 is continuous but not decreasing. The indifference boundary in Fig. 3 is neither continuous nor monotone. Now we show that under Condition 1, we can translate a wealth-based policy $t$ to a benefit-based policy $s$ in such a way that the assignment sets under the two policies are identical. ${ }^{8}$

Condition 1 implies that the inverse of $\theta$ exists for the set of benefits $\left[\ell^{\prime}, \bar{\ell}^{\prime}\right] \equiv$ $\theta([\underline{w}, \bar{w}])$, the range of the function $\theta$. Let this inverse be $\phi:\left[\underline{\ell}^{\prime}, \bar{\ell}^{\prime}\right] \rightarrow[\underline{w}, \bar{w}]$. For any benefit $\ell$ in $\left[\underline{\ell}^{\prime}, \bar{\ell}^{\prime}\right]$, the function $\phi$ gives the wealth level at which the consumer will be just willing to pay $t(w)$ to purchase the good. Note that under Condition 1 , $\theta(\underline{w})=\bar{\ell}^{\prime}>\theta(\bar{w})=\underline{\ell}^{\prime}$.

The range of $\theta,\left[\underline{\ell}^{\prime}, \bar{\ell}^{\prime}\right]$, need not be exactly $[\underline{\ell}, \bar{\ell}]$, but because the assignment set $\alpha(t)$ is nonempty and a proper subset of all consumers, it must intersect $[\underline{\ell}, \bar{\ell}]$. The next two diagrams illustrate two possibilities. In Fig. 4, the range of $\theta$ contains $[\underline{\ell}, \bar{\ell}]$, while in Fig. 5, the range of $\theta$ is a proper subset of $[\underline{\ell}, \bar{\ell}]$.

Now we construct a benefit-based policy, $s:[\underline{\ell}, \bar{\ell}] \rightarrow \mathbb{R}^{+}$, which yields the same indifference boundary as $t$. For each $\ell \in[\underline{\ell}, \bar{\ell}] \cap\left[\underline{\ell}^{\prime}, \bar{\ell}^{\prime}\right]$, we define a payment $s(\ell)$ by

$$
U(\phi(\ell)-s)+\ell=U(\phi(\ell)) .
$$

We replace the wealth variable in the definition of the indifference boundary (7) by $\phi(\ell)$. The equation in (10) yields an implicit function $s(\ell)$, a benefit-based policy. The construction of such an $s(\ell)$ yields an identical boundary: $U(w-s(\ell))+\ell=U(w)$, but now the policy defined by (10) is written in terms of benefits instead of wealth. Consumer type $(w, \theta(w))$ is just willing to pay $t(w)$ to get the good for the benefit $\theta(w)$ if and only if consumer type $(\phi(\ell), \ell)$ is just willing to pay $s(\ell)=t(w)$ to get the good for the benefit $\ell$.

There remain possible values of benefits which are not in the range $\left[\underline{\ell}^{\prime}, \bar{\ell}^{\prime}\right]$. These cases, when they exist, correspond to $\underline{\ell}<\underline{\ell}^{\prime}, \bar{\ell}^{\prime}<\bar{\ell}$, or both (see Figs. 4, 5). We complete the definition of $s$ by the following.

$$
\text { For } \ell \in\left[\underline{\ell}, \underline{\ell}^{\prime}\right] \text {, let } s(\ell)=s\left(\underline{\ell}^{\prime}\right) \text {. For } \ell \in\left[\bar{\ell}^{\prime}, \bar{\ell}\right] \text {, let } s(\ell)=s\left(\bar{\ell}^{\prime}\right) \text {. }
$$

\footnotetext{
${ }^{8}$ For a general utility function as in Footnote 5, an indifference boundary is implicitly defined by $U(w-$ $t(w), \ell)=U(w, 0)$. The condition here (and those to be presented later) will then refer to the monotonicity of this boundary.
} 
Fig. $4\left[\underline{\ell}^{\prime}, \bar{\ell}^{\prime}\right]$ contains $[\underline{\ell}, \bar{\ell}]$

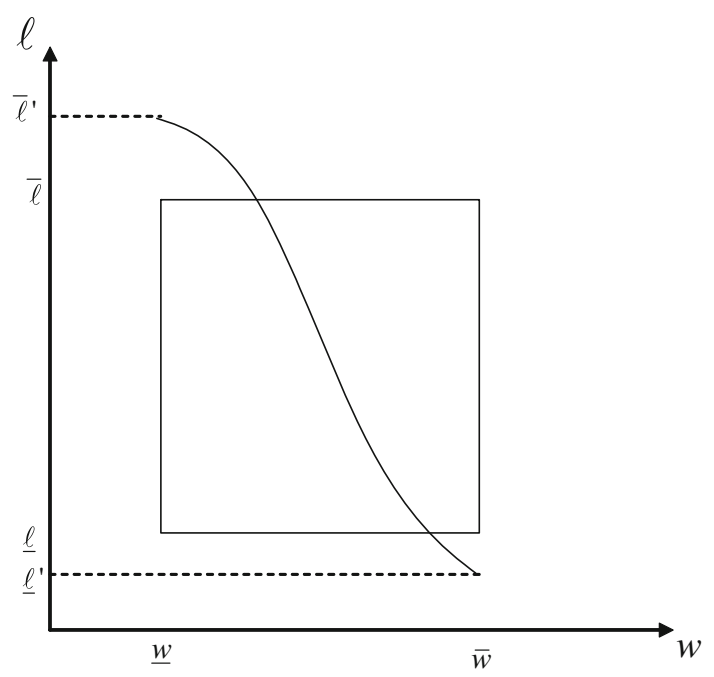

Fig. $5[\underline{\ell}, \bar{\ell}]$ contains $\left[\underline{\ell}^{\prime}, \bar{\ell}^{\prime}\right]$

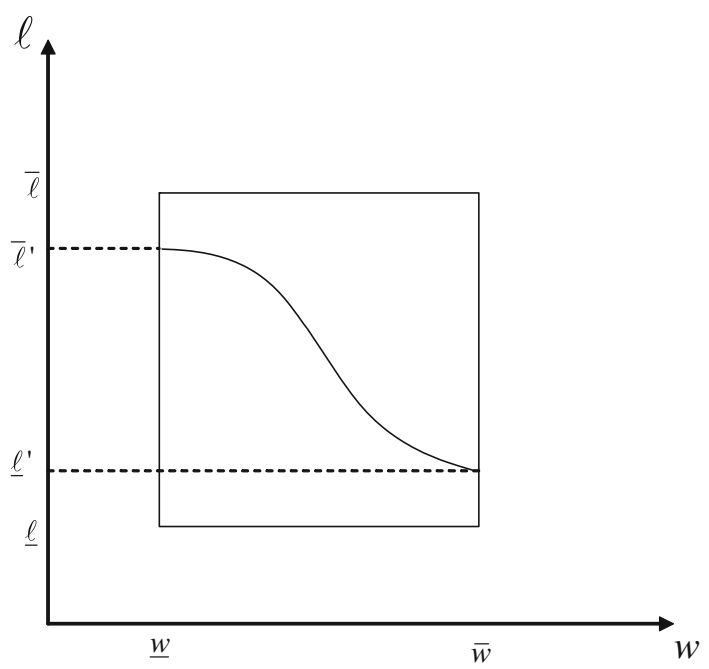

The two sets, $\left[\underline{\ell}, \underline{\ell}^{\prime}\right]$ and $\left[\bar{\ell}^{\prime}, \bar{\ell}\right]$, contain consumers with very low or very high benefits. Under $t(w)$, those consumers with very low benefits will not purchase the good at $s\left(\underline{\ell}^{\prime}\right)$ no matter how high their wealth $w$; those with very high benefits will always purchase at $s\left(\bar{\ell}^{\prime}\right)$. This completes the translation of a wealth-based policy $t(w)$ to a benefit-based policy $s(\ell)$.

Proposition 1 Suppose that a wealth-based policy $t:[\underline{w}, \bar{w}] \rightarrow \mathbb{R}^{+}$satisfies Condition 1 (decreasing indifference boundary). The benefit-based policy $s:[\underline{\ell}, \bar{\ell}] \rightarrow \mathbb{R}^{+}$ defined in (10) and (11) implements the same assignment as the wealth-based policy $t$. That is, assignment sets $\alpha(t)$ and $\beta(s)$ are identical. 
Fig. 6 Downward sloping boundary: direction of preferences

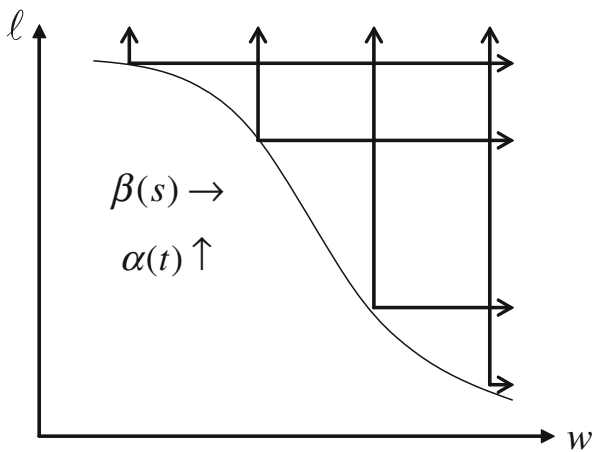

Proposition 1 (whose proof is in the Appendix) makes use of the decreasing monotonicity of the indifference boundary. Given a wealth-based policy, to each wealth level, we associate a benefit threshold at which the consumer is indifferent between purchasing and not. The strict monotonicity of the boundary allows us to invert this relationship. So now for each benefit level, we are able to associate a wealth threshold.

Monotonicity alone does not guarantee that the assignments are identical when a wealth-based policy is translated to a benefit-based policy. An indifference boundary divides the space of all consumers into two half spaces. Consumers' preferences determine in which one of these half spaces the assignment set resides. Given a boundary $\theta$ in $w-\ell$ space, at a point $(w, \ell)$ on the boundary, by (2), those points above $(w, \ell)$ are consumers with higher benefits, and belong to the set $\alpha(t)$. Conversely, given the equivalent boundary $\phi$ (the inverse of $\theta$ ) on the same $w-\ell$ space, then at a point $(w, \ell)$ on the boundary, those points to the right of $(w, \ell)$ are consumers with higher wealth, and belong to the set $\beta(s)$. Figure 6 illustrates this. When an indifference boundary is strictly decreasing, the assignment sets of the wealth-based and translated benefit-based policies coincide. In the Appendix, we present Example 1, which uses a logarithmic utility function to show explicitly the translation of a wealth-based policy to a benefit-based policy.

Corollary 1 Suppose that a wealth-based policy t satisfies Condition 1 (decreasing indifference boundary) and is increasing. The assignment-equivalent benefit-based policy s is decreasing in $\ell$.

Consider two types of consumers $\left(w_{1}, \ell_{2}\right)$ and $\left(w_{2}, \ell_{1}\right)$ who are on the indifference boundary under $t$ and the equivalent $s$. Without loss of generality, let $w_{1}<w_{2}$. Because the indifference boundary is decreasing, $\ell_{1}<\ell_{2}$; see Fig. 7. By the assumption in Corollary $1, t\left(w_{1}\right)<t\left(w_{2}\right)$. Under the equivalent benefit-based policy $s$, type $\left(w_{1}, \ell_{2}\right)$ pays $s\left(\ell_{2}\right)=t\left(w_{1}\right)$, and type $\left(w_{2}, \ell_{1}\right)$ pays $s\left(\ell_{1}\right)=t\left(w_{2}\right)$. It follows that $s\left(\ell_{2}\right)<s\left(\ell_{1}\right)$. Corollary 1 illustrates an interesting implication of our method. Suppose that a regulator makes richer consumers pay more for the good. If this policy $t$ generates a decreasing indifference boundary, then the assignment-equivalent benefit-based policy will make more deserved consumers pay less.

Although a consumer makes the same purchase decision under equivalent subsidy schemes, he generally obtains different utilities from them, except when his type is 


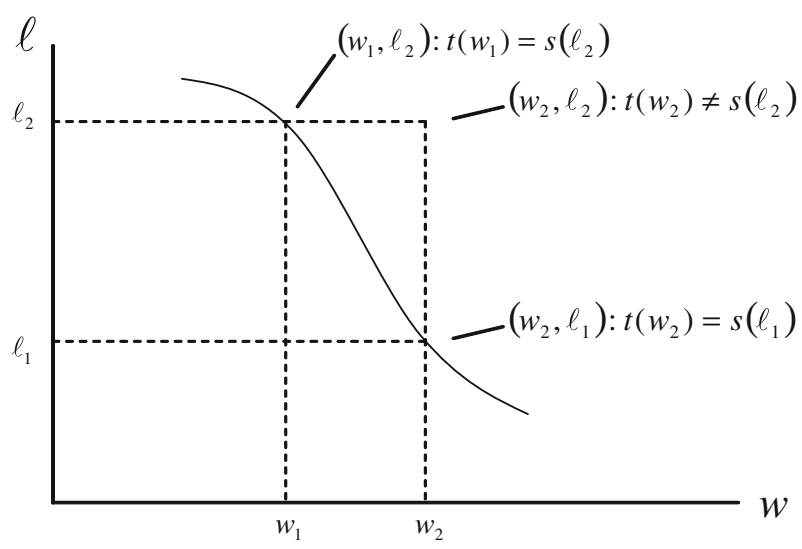

Fig. 7 Types of consumers on a decreasing indifference boundary

on the indifference boundary. A consumer's surplus from the subsidy can be measured by how far away he is from the indifference boundary. Consider consumer $\left(w_{2}, \ell_{2}\right)$ in Fig. 7. Under a wealth-based subsidy, this consumer obtains a surplus $U\left(w_{2}-t\left(w_{2}\right)\right)+\ell_{2}-U\left(w_{2}\right)=\ell_{2}-\ell_{1}$ because $U\left(w_{2}\right)=U\left(w_{2}-t\left(w_{2}\right)\right)+\ell_{1}$. The surplus is increasing in $\ell_{2}-\ell_{1}$, the distance between type $\left(w_{2}, \ell_{2}\right)$ and the boundary in the $\ell$-direction. Under the equivalent benefit-based subsidy, the consumer obtains a surplus $U\left(w_{2}-s\left(\ell_{2}\right)\right)+\ell_{2}-U\left(w_{2}\right)=\left[U\left(w_{2}-s\left(\ell_{2}\right)\right)-U\left(w_{1}-s\left(\ell_{2}\right)\right)\right]-\left[U\left(w_{2}\right)-U\left(w_{1}\right)\right]$ because $U\left(w_{1}-s\left(\ell_{2}\right)\right)+\ell_{2}=U\left(w_{1}\right)$. Since $U$ is strictly concave, this surplus is increasing in $w_{2}-w_{1}$, the distance between type $\left(w_{2}, \ell_{2}\right)$ and the boundary in the $w$-direction. Consumers who are near the indifference boundary in one dimension, but far from it in the other dimension may stand to gain or lose more under equivalent subsidy schemes.

In the Introduction, we have mentioned cost-effectiveness and means-testing as common subsidy schemes. In a typical cost-effectiveness allocation, a consumer receives a good when the benefit-cost ratio is sufficiently high. In our context, since the cost $c$ is constant, cost-effectiveness says that those with benefits above a threshold, say $\tilde{\ell}$, should consume the good. The corresponding assignment set will be the half space above the horizontal line at $\widetilde{\ell}$ in the $w-\ell$ space of consumers. This horizontal line is like the indifference boundary. Condition 1 is not satisfied for a horizontal indifference boundary. However, we can implement an assignment that is arbitrarily close to the cost-effectiveness assignment.

Let $t$ be a differentiable wealth-based subsidy. Next we choose $\varepsilon>0$ but arbitrarily small, and set the slope of the indifference boundary, from (9), to:

$$
\frac{\mathrm{d} \hat{\ell}}{\mathrm{d} w}=\left[U^{\prime}(w)-U^{\prime}(w-t(w))\right]+U^{\prime}(w-t(w)) t^{\prime}(w)=-\varepsilon<0
$$

Now Condition 1 is satisfied. Equation (12) is a first-order differential equation in $t$. We can set the initial condition $t(\underline{w})$ so that $U(\underline{w}-t(\underline{w}))+\tilde{\ell}=U(\underline{w})$. Then the solution will yield an indifference boundary that is almost horizontal, and arbitrarily near 
Fig. 8 Upward sloping boundary: direction of preferences

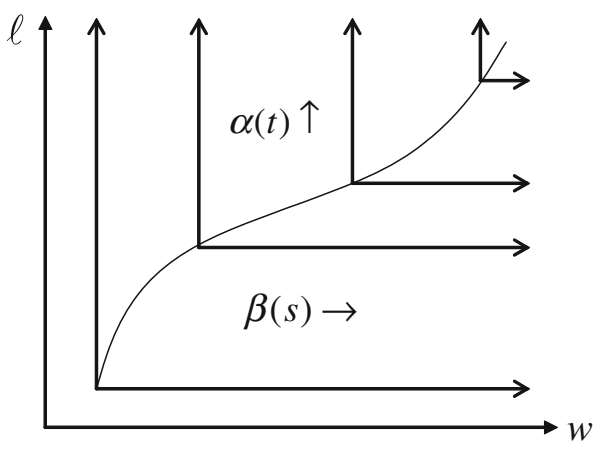

the cost-effectiveness boundary (a horizontal line at $\widetilde{\ell}$ ). Furthermore, $t^{\prime}(w)>0$ so that richer consumers pay more. To see this, consider a consumer with wealth slightly more than $\underline{w}$. If $t(w)$ remains at $t(\underline{w})$, then $t^{\prime}(w)=0$, the slope of the indifference boundary in (12) becoming strictly negative. This violates the requirement that the slope must be arbitrarily small, so $t$ must increase with $w$.

We have implemented cost-effectiveness assignment sets approximately with wealth information. Since the wealth-based subsidy satisfies Condition 1, we can also translate it to an assignment-equivalent benefit-based subsidy. Our results therefore draw a connection between means-test programs such as Medicaid in the U.S. and cost-effectiveness national health services in Canada and Europe.

The indifference boundary defined by (12) is almost flat. Under wealth-based subsidies, consumer $(w, \ell)$ has a surplus proportional to $\ell-\widetilde{\ell}$, and this is almost independent of $w$. Implementation of cost-effectiveness by wealth subsidies actually implies that poor and rich consumers benefit more or less by the same amount. By contrast, under benefit-based subsidies, consumer $(w, \ell)$, in addition to the surplus $\ell-\widetilde{\ell}$, has an extra surplus proportional to $w-\underline{w}$, so richer consumers gain more.

We next consider the case when the indifference boundary is strictly increasing. Then the union of the assignment sets $\alpha(t)$ and $\beta(s)$ of equivalent boundaries is the set of all consumers and the intersection contains only the indifference boundary. Figure 8 illustrates the direction of the preferences when the boundary is upward sloping.

Condition 2 (Increasing indifference boundary) The wealth-based policy $t$ is continuous (equivalently the function $\theta$ is continuous). The indifference boundary $\theta$ is strictly increasing.

Corollary 2 Suppose that a wealth-based policy $t$ satisfies Condition 2 (increasing indifference boundary). The benefit-based policy s defined in (10) and (11) implements an assignment $\beta(s)$ whose intersection with the assignment $\alpha(t)$ implemented by the wealth-based policy $t(w)$ is the set of indifferent consumers $\{(w, \ell): U(w-t(w))+$ $\ell=U(w)\}$, and whose union with $\alpha(t)$ is the set of all consumers $[\underline{w}, \bar{w}] \times[\underline{\ell}, \bar{\ell}]$.

We omit the proof; it is symmetric to the proof of Proposition 1.

We now briefly describe the translation of a benefit-based policy to a wealthbased policy since the formal steps are similar. Given a policy $s:[\underline{\ell}, \bar{\ell}] \rightarrow \mathbb{R}^{+}$, 
an indifference boundary $\varphi:[\underline{\ell}, \bar{\ell}] \rightarrow \mathbb{R}$ is defined by

$$
\varphi(\ell)=\hat{w} \quad \text { where } U(\hat{w}-s(\ell))+\ell=U(\hat{w})
$$

and now we can state the corresponding condition:

Condition 3 (Decreasing indifference boundary) The benefit-based policy s is continuous (equivalently the function $\varphi$ is continuous). The indifference boundary $\varphi$ is strictly decreasing.

Under Condition 3, let the inverse of $\varphi$ be $\vartheta:\left[\underline{w}^{\prime}, \bar{w}^{\prime}\right] \rightarrow[\underline{\ell}, \bar{\ell}]$, where $\left[\underline{w}^{\prime}, \bar{w}^{\prime}\right]$ is the range of $\varphi$. Then we use the same steps (see (11) above) to assign values to $\vartheta$ for those $w$ outside of $\left[\underline{w}^{\prime}, \bar{w}^{\prime}\right]$, the range of $\varphi$. Now we construct a wealth-based policy $t:[\underline{w}, \bar{w}] \rightarrow \mathbb{R}^{+}$by

$$
U(w-t)+\vartheta(w)=U(w)
$$

Proposition 2 Suppose that a benefit-based policy $s:[\underline{\ell}, \bar{\ell}] \rightarrow \mathbb{R}^{+}$satisfies Condition 3 (decreasing indifference boundary). The wealth-based policy $t:[\underline{w}, \bar{w}] \rightarrow \mathbb{R}^{+}$ defined in (14) implements the same assignment as the benefit-based policy s. That is, the two sets $\alpha(t)$ and $\beta(s)$ are identical.

Versions of Corollaries 1 and 2 hold for the policy $s$ and the translated policy $t$. In the Appendix, Example 2 presents explicitly the translation of a benefit-based policy to a wealth-based policy for a logarithmic utility function.

\subsection{Implementable assignments}

In this subsection, we characterize assignment sets that are implementable by wealthbased policies, benefit-based policies, and both simultaneously. Let $\Omega$ be a nonempty and proper subset of $[\underline{w}, \bar{w}] \times[\underline{\ell}, \bar{\ell}]$. The set $\Omega$ is said to be implementable by a wealthbased policy if there exists $t:[\underline{w}, \bar{w}] \rightarrow \mathbb{R}^{+}$such that $\Omega=\{(w, \ell): U(w-t(w))+$ $\ell \geq U(w)\}$. Similarly, the set $\Omega$ is said to be implementable by a benefit-based policy if there exists $s:[\underline{\ell}, \bar{\ell}] \rightarrow \mathbb{R}^{+}$such that $\Omega=\{(w, \ell): U(w-s(\ell))+\ell \geq U(w)\}$. Finally, the set $\Omega$ is said to be simultaneously implementable by a wealth-based policy and a benefit-based policy if there exist $t:[\underline{w}, \bar{w}] \rightarrow \mathbb{R}^{+}$and $s:[\underline{\ell}, \bar{\ell}] \rightarrow \mathbb{R}^{+}$such that $\Omega=\{(w, \ell): U(w-t(w))+\ell \geq U(w)\}=\{(w, \ell): U(w-s(\ell))+\ell \geq U(w)\}$.

We use the monotonicity properties in (2) to obtain some characterization of implementable sets. Suppose that $\Omega$ is implementable by a wealth-based policy. So there is $t(w)$ such that $U(w-t(w))+\ell \geq U(w)$ for $(w, \ell) \in \Omega$. For any $\ell^{\prime}>\ell$, we have $U(w-t(w))+\ell^{\prime}>U(w)$; if $(w, \ell) \in \Omega,\left(w, \ell^{\prime}\right) \in \Omega$. Similarly, suppose that $\Omega$ is implementable by a benefit-based policy, and $(w, \ell) \in \Omega$, then $\left(w^{\prime}, \ell\right) \in \Omega$ whenever $w^{\prime}>w$ because $U(w-s(\ell))+\ell \geq U(w)$ implies $U\left(w^{\prime}-s(\ell)\right)+\ell \geq U\left(w^{\prime}\right)$. Figure 3 above illustrates a set that is implementable by a wealth-based policy but not by a benefit-based policy. 
The following two lemmas characterize assignment sets that are simultaneously implementable by wealth-based and benefit-based policies. Their proofs are in Appendix.

Lemma 1 Let $\Omega$ be simultaneously implementable by a wealth-based policy and a benefit-based policy. Then $\Omega$ is a closed set, and the wealth-based and benefit-based policies that implement $\Omega$ are continuous.

Intuitively, if $\Omega$ is implementable by a wealth-based policy, we have $\Omega=\{(w, \ell)$ : $U(w-t(w))+\ell \geq U(w)\}$, for some $t$. Now the utility $U(w-t(w))+\ell$ is continuous in $\ell$. So if we consider a sequence $\left(w, \ell_{i}\right) \in \Omega$, and if the limit of $\ell_{i}$ is $\ell$, then $(w, \ell)$ must also belong to $\Omega$ by continuity. We can repeat the same argument for a similar sequence $\left(w_{i}, \ell\right)$ when $\Omega$ is implementable by a benefit-based policy. It follows that if $\Omega$ is simultaneously implementable, any converging sequence $\left(w_{i}, \ell_{i}\right)$ must have a limit in $\Omega$ if all elements of the sequence belong to $\Omega$.

Lemma 2 Let $\Omega$ be simultaneously implementable by a wealth-based policy and a benefit-based policy. The indifference boundary, $\ell$ as a function of $w$ or vice versa, defined implicitly by either $U(w-t(w))+\ell=U(w)$ or $U(w-s(\ell))+\ell=U(w)$, must be strictly decreasing.

Intuitively, the utility $U(w-t(w))+\ell$ is strictly increasing in $\ell$ when $\Omega$ is implementable by a wealth-based policy, and the utility $U(w-s(\ell))+\ell$ is strictly increasing in $w$ when $\Omega$ is implementable by a benefit-based policy. So when $\Omega$ is simultaneously implementable, the boundary cannot remain constant over an interval of $w$ or $\ell$. From Fig. 8 wealth-based and subsidy-based schemes that give rise to a strictly increasing boundary never induce the same assignment set. Hence, the indifference boundary must be strictly decreasing if the assignment set is simultaneously implementable. Lemmas 1 and 2 together establish the following:

Proposition 3 If $\Omega$ is implementable simultaneously by wealth-based policy $t:[\underline{w}$, $\bar{w}] \rightarrow \mathbb{R}^{+}$and benefit-based policy $s:[\underline{\ell}, \bar{\ell}] \rightarrow \mathbb{R}^{+}$, each of these policies must be continuous, and each must induce a decreasing indifference boundary.

Conditions 1 and 3 are necessary and sufficient for an assignment set to be implemented by both wealth-based and benefit-based policies. Observing either wealth or benefit information is sufficient for the implementation of an assignment set with a decreasing indifference boundary. Conversely, when the indifferent boundary of an assignment set is not decreasing, the implementation of the assignment set requires specific information. For example, if the indifference boundary is U-shaped in $w-\ell$ space, or like the one in Fig. 3, then wealth information is required, but no subsidy scheme based on benefit can implement the assignment.

\section{Equivalent policies and revenues}

In Sect. 3, we relate the wealth-based and benefit-based policies that implement the same assignment. The required budgets (see (5) and (6)) for each of the two equivalent 
sets are different. Consider the three consumer types in Fig. 7: $\left(w_{1}, \ell_{2}\right),\left(w_{2}, \ell_{1}\right)$, and $\left(w_{2}, \ell_{2}\right)$, with $w_{1}<w_{2}$ and $\ell_{1}<\ell_{2}$. Let $t:[\underline{w}, \bar{w}] \rightarrow \mathbb{R}^{+}$and $s:[\underline{\ell}, \bar{\ell}] \rightarrow \mathbb{R}^{+}$ be the equivalent subsidy schemes. By construction, we have $t\left(w_{1}\right)=s\left(\ell_{2}\right)$, and $t\left(w_{2}\right)=s\left(\ell_{1}\right)$. Unless $t$ and $s$ are constant functions, $t\left(w_{2}\right) \neq t\left(w_{1}\right)=s\left(\ell_{2}\right)$, so the regulator collects different revenues from equivalent policies.

Proposition 4 (Revenue nonequivalence) If a wealth-based policy $t:[\underline{w}, \bar{w}] \rightarrow \mathbb{R}^{+}$ and a benefit-based policy $s:[\underline{\ell}, \bar{\ell}] \rightarrow \mathbb{R}^{+}$implement the same assignment, they generate different revenues (and therefore require different budgets) for generic distributions of wealth $(F(w))$ and benefits $(G(\ell))$ except when $t(w)=s(\ell)=k, a$ constant.

In general, it is not possible to rank revenues according to only properties of $F$ and $G$. The reason is that the assignment set is defined with respect to the policies without any reference to these distributions. Example 3, in the Appendix, shows a large difference (in percentage) of revenues collected by the regulator under wealth-based and benefit-based policies. In turn these policies imply a large difference of required budgets to implement the same assignment set.

Proposition 4 has a straightforward policy implication. Suppose that the regulator has to decide which information, wealth or benefits, needs to be collected. The total cost for an information regime is the sum of the required budget and the information collection cost. Cost savings for the implementation of a given assignment set can be achieved by selecting the policy that requires a smaller total cost.

\subsection{Equivalent revenue and general subsidy}

We have assumed up to now that a consumer makes a payment to the regulator only when he purchases the good. Now, we allow the regulator to impose a tax or subsidy to consumers who decide not to purchase. This can be regarded as a general taxation scheme which consumers cannot opt out of. We show that then equivalent benefit-based and wealth-based policies may be chosen to generate the same revenue.

We will only consider the case of wealth-based subsidies; the case of benefitbased subsidies is similar. A wealth-based policy is now a pair of payment functions $\left[t_{1}:[\underline{w}, \bar{w}] \rightarrow \mathbb{R}, t_{2}:[\underline{w}, \bar{w}] \rightarrow \mathbb{R}\right]$; a consumer with wealth $w$ pays $t_{1}(w)$ when he does not purchase the good, and $t_{2}(w)$ when he does. We allow the values $t_{1}(w)$ and $t_{2}(w)$ to be positive or negative. For a given policy $\left(t_{1}, t_{2}\right)$, we define the assignment set:

$$
\alpha\left(t_{1}, t_{2}\right) \equiv\left\{(w, \ell): U\left(w-t_{2}(w)\right)+\ell \geq U\left(w-t_{1}(w)\right)\right\}
$$

and the revenue collected is:

$$
R\left(t_{1}, t_{2}\right) \equiv \int_{\alpha\left(t_{1}, t_{2}\right)^{c}} t_{1}(w) \mathrm{d} F(w) \mathrm{d} G(\ell)+\int_{\alpha\left(t_{1}, t_{2}\right)} t_{2}(w) \mathrm{d} F(w) \mathrm{d} G(\ell)
$$


(The superscript $c$ over the sets $\alpha\left(t_{1}, t_{2}\right)$ and $\beta\left(s_{1}, s_{2}\right)$ below, denotes their complements.)

The equation $U\left(w-t_{2}(w)\right)+\ell=U\left(w-t_{1}(w)\right)$ defines a relationship between benefit $\ell$ and wealth $w$, and generates the indifference boundary $\ell=\theta\left(w ; t_{1}, t_{2}\right)$. When $\left[t_{1}, t_{2}\right]$ is continuous and $\theta\left(w ; t_{1}, t_{2}\right)$ strictly decreasing in $w$, Proposition 1 applies. There is a benefit-based policy $\left[s_{1}:[\underline{\ell}, \bar{\ell}] \rightarrow \mathbb{R}, s_{2}:[\underline{\ell}, \bar{\ell}] \rightarrow \mathbb{R}\right]$ implementing the same assignment set, $\beta\left(s_{1}, s_{2}\right)=\alpha\left(t_{1}, t_{2}\right)$, where $s_{1}(\ell)$ is the payment by a consumer with benefit $\ell$ when he does not purchase, and $s_{2}(\ell)$ is the payment when he does. The construction of the benefit-based policy uses the same procedure. Let $\phi$ be the inverse of the (strictly decreasing) indifference boundary $\theta$. For each $\ell$ we choose $s_{1}(\ell)$ and $s_{2}(\ell)$ to satisfy

$$
U\left(\phi(\ell)-s_{2}(\ell)\right)+\ell=U\left(\phi(\ell)-s_{1}(\ell)\right)
$$

Many benefit-based policies satisfy (17). The revenue collected under $\left[s_{1}, s_{2}\right]$ is

$$
R\left(s_{1}, s_{2}\right) \equiv \int_{\beta\left(s_{1}, s_{2}\right)^{c}} s_{1}(\ell) \mathrm{d} F(w) \mathrm{d} G(\ell)+\int_{\beta\left(s_{1}, s_{2}\right)} s_{2}(\ell) \mathrm{d} F(w) \mathrm{d} G(\ell)
$$

Proposition 5 (Revenue equivalence) Suppose that a wealth-based policy $\left[t_{1}, t_{2}\right]$ satisfies Condition 1 (decreasing indifference boundary). There exists a benefit-based policy $\left[s_{1}, s_{2}\right]$ that implements the same assignment set and generates the same revenue: $\alpha\left(t_{1}, t_{2}\right)=\beta\left(s_{1}, s_{2}\right)$ and $R\left(t_{1}, t_{2}\right)=R\left(s_{1}, s_{2}\right)$.

The intuition for Proposition 5 (whose proof is in the Appendix) is best illustrated by a two-part tariff policy. Given a wealth-based policy $\left[t_{1}, t_{2}\right]$, let $\left[s_{1}, s_{2}\right]$ be an equivalent benefit-based policy. There are many such policies. Let $s_{1}(\ell)=M$, a constant, and $s_{2}(\ell)=M+s(\ell)$, where $M$ can be regarded as a lump sum tax or subsidy for each consumer, and $s(\ell)$ is the incremental payment for purchasing the good. The revenue that is collected by $\left[s_{1}(\ell), s_{2}(\ell)\right]=[M, M+s(\ell)]$ is now

$$
M+\int_{\beta(M, M+s)} s(\ell) \mathrm{d} F(w) \mathrm{d} G(\ell)
$$

The level of $M$ uses up one degree of freedom in the choice of the benefit-based policy $\left[s_{1}(\ell), s_{2}(\ell)\right]$. For each given value of $M$, we can set $s(\ell)$ to satisfy

$$
U(\phi(\ell)-M-s(\ell))+\ell=U(\phi(\ell)-M)
$$

maintaining the same assignment set. We can adjust the level of $M$ to achieve $R(M$, $M+s)=R\left(t_{1}, t_{2}\right)$, and the same revenue will be collected.

Proposition 5 does not say that consumers derive the same utility whether they are subsidized and taxed according to their wealth or benefit. Nevertheless, suppose that the social objective is focused on an assignment set. If this assignment has a strictly 
decreasing boundary, it is implementable simultaneously by wealth-based and benefit-based subsidies. Proposition 5 then says that the total subsidy required will be the same for these policies. The deciding factor becomes the relative cost of collecting and processing the wealth and benefit information.

\section{Optimal subsidy and indifference boundary}

In this section, we characterize an optimal subsidy policy. Here we adopt a conventional approach, employing a utilitarian social welfare function for the analysis. We consider only wealth-based subsidies. A total budget $B$ is to be allocated to consumers. A subsidy policy is a function $t:[\underline{w}, \bar{w}] \rightarrow \mathbb{R}^{+}$.

Given a subsidy policy, those consumers with wealth $w$ prefer to purchase the good if and only if their benefits are above a threshold $\hat{\ell}(w)$ given by:

$$
U(w-t(w))+\hat{\ell}=U(w) .
$$

This threshold $\hat{\ell}(w)$ is the indifference boundary we have discussed in the previous sections. The mass of consumers with wealth $w$ who purchase is $[1-G(\hat{\ell})]$. Each of these consumers pays $t(w)$ while the regulator pays the balance $c-t(w)$. Out of the budget $B$ consumers with wealth $w$ use $[1-G(\hat{\ell})][c-t(w)]$. Therefore, the budget constraint is

$$
\int_{\underline{w}}^{\bar{w}}[1-G(\hat{\ell})][c-t(w)] \mathrm{d} F(w)=B .
$$

The regulator maximizes a utilitarian social welfare function. An optimal wealthbased policy is $t(w)$ and the associated $\hat{\ell}(w)$ that maximize

$$
\int_{\underline{w}}^{\bar{w}}\left\{\int_{\underline{\ell}}^{\widehat{\ell}} U(w) \mathrm{d} G(\ell)+\int_{\widehat{\ell}}^{\bar{\ell}}[U(w-t(w))+\ell] \mathrm{d} G(\ell)\right\} \mathrm{d} F(w)
$$

subject to (21) and (22). In the objective function above, for each wealth level $w$, consumers with benefit below $\hat{\ell}(w)$ do not buy, and their utility is given by the integral of $U(w)$ from $\underline{\ell}$ to $\widehat{\ell}$, while those consumers with benefit above $\hat{\ell}(w)$ buy and their utility is given by the integral of $U(w-t(w))+\ell$ from $\widehat{\ell}$ to $\bar{\ell}$.

From the constraint (21), we obtain $U(w-t(w))=U(w)-\hat{\ell}$, which can be substituted into the objective function. Furthermore, from constraint (21) $t(w)=$ $w-h(U(w)-\hat{\ell})$, where $h \equiv U^{-1}$, the inverse of $U$. We substitute for $t(w)$ in (22) to obtain an equivalent constrained optimization program: choose $\widehat{\ell}(w)$ to maximize

$$
\int_{\underline{w}}^{\bar{w}}\left\{U(w)+\int_{\widehat{\ell}}^{\bar{\ell}}[\ell-\widehat{\ell}] \mathrm{d} G(\ell)\right\} \mathrm{d} F(w)
$$


subject to

$$
\int_{\underline{w}}^{\bar{w}}[1-G(\hat{\ell})][c-w+h(U(w)-\hat{\ell})] \mathrm{d} F(w)=B .
$$

We use pointwise optimization to characterize the optimal threshold, but will not present the first-order conditions here.

The optimal threshold and subsidy follow the following equation:

$$
1=\lambda\left[\frac{g(\widehat{\ell})}{1-G(\widehat{\ell})}[c-t(w)]+\frac{1}{U^{\prime}(w-t(w))}\right]
$$

where $\lambda>0$ is the multiplier for the constraint (25). Because $\lambda$ is a constant, the total derivative of the term in the square brackets in (26) must be zero. So we can obtain an expression for the derivative of $\widehat{\ell}$ with respect to $w$, which is the slope of the indifference boundary (this expression is available from the authors). This derivative may be positive or negative depending on the value of $w$. It is quite possible that the optimal wealth-based policy induces a nonmonotone indifference boundary. We provide some interpretations of these possibilities now.

Instead of using a policy $t(w)$ as a choice instrument, we can let the regulator decide on a budget allocation rule as a function of wealth. Let this be $B(w)$, the resource allocated to those consumers with wealth $w$. If the resource $B(w)$ is to be exhausted by these consumers with wealth $w$, the regulator must consider a threshold and a payment, $\widehat{\ell}$ and $t$, such that

$$
\begin{gathered}
U(w-t)+\hat{\ell}=U(w) \\
{[1-G(\hat{\ell})][c-t]=B(w)}
\end{gathered}
$$

which are similar to (21) and (22) above. A payment policy is equivalent to a budget allocation rule. We have seen that an optimal policy may give rise to a nonmonotone indifference boundary. This can also be understood in terms of the properties of the optimal budget allocation rule.

Assume that $B(w)$ is differentiable. From the implicit function theorem, $\widehat{\ell}$ and $t$ defined in (27) and (28) can be regarded as functions of $w$. Again, the function $\widehat{\ell}(w)$ is the indifference boundary. After total differentiation, we obtain

$$
\frac{\mathrm{d} \widehat{\ell}}{\mathrm{d} w}=-\frac{U^{\prime}(w-t) B^{\prime}(w)+[1-G(\widehat{\ell})]\left[U^{\prime}(w-t)-U^{\prime}(w)\right]}{1-G(\widehat{\ell})+g(\widehat{\ell}) U^{\prime}(w-t)(c-t)} .
$$

The indifference boundary is negatively sloped if $B(w)$ is (weakly) increasing. That is, if the optimal allocation rule sets a budget (weakly) increasing in wealth, then the indifference boundary is strictly decreasing.

Figure 9 illustrates this property. The upward sloping lines are Eq. (27) for $w=w_{1}$ and $w_{2}$, while the downward sloping lines are Eq. (28) for $w=w_{1}$ and $w_{2}$. An intersection point represents a pair of $\hat{\ell}$ and $t$ satisfying both (27) and (28). Figure 9 shows 


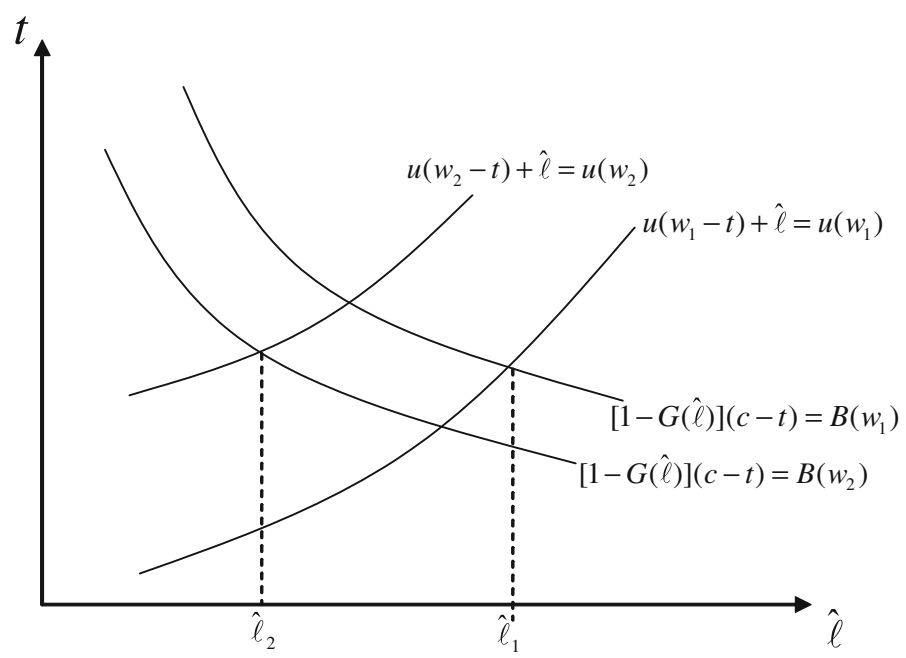

Fig. 9 Budget and benefit threshold for consumers with wealth $w$

two intersection points, $\hat{\ell}_{1}$ and $\hat{\ell}_{2}$. An increase in $w$ shifts Eq. (27) upward because $U$ is concave. If $B(w)$ is increasing in $w$, then an increase in $w$ shifts Eq. (28) downward. The intersection $\hat{\ell}_{2}$ must then moves to the left of $\hat{\ell}_{1}$. That is, $\hat{\ell}$ is decreasing in $w$, or the indifference boundary is decreasing. If, however, $B(w)$ is decreasing, the downward sloping equation (28) may shift upward when $w$ increases. In this case, the intersection point may well move to the right of $\hat{\ell}_{1}$ as $w$ increases, and the indifference boundary may not be decreasing.

Proposition 6 Suppose that a regulator sets a wealth-based policy $t(w)$, or equivalently, a budget allocation policy $B(w)$ for consumers with wealth $w$, to maximize a social welfare function. Suppose that the optimal budget allocation policy is increasing in w. Then the optimal wealth-based policy must give rise to a strictly decreasing indifference boundary.

For a utilitarian social welfare function, an optimal policy $t(w)$ may well be consistent with a decreasing indifference boundary. For some other social welfare functions, the optimal wealth-based policy may also induce a decreasing indifference boundary. Our study on assignment sets and the policies that implement them complements the conventional welfare analysis.

\section{Conclusion}

We offer a new perspective on subsidy policies for an indivisible good. Our approach does not impose optimality properties on subsidy schemes, as they may have originated from historical, political, or sociological considerations. Instead, we translate subsidy policies based on wealth to those based on benefit, and vice versa. Under a continuity-monotonicity condition, these translated wealth-based and benefit-based 
schemes implement the same assignment of the good to consumers. This continuitymonotonicity condition is also necessary for any assignment set to be implemented simultaneously by wealth-based and benefit-based schemes. In any setting where consumers' willingness to pay for an indivisible good depends on two dimensions, the translation method can be applied.

We show that equivalent subsidy schemes require different budgets to support the same assignment set unless general taxes or subsidies (such as lump sum or poll taxes) are available. However, when general taxes or subsidies are infeasible, we provide a method to compare the implementation cost of equivalent subsidy schemes. One can also include for comparison the cost of collecting information on wealth and on benefits. By adding the information collection cost to the implementation cost one obtains the total costs of subsidy schemes for a given assignment set.

Our approach, however, necessarily yields a sort of partial ordering. Since we do not postulate a social welfare objective, we are unable to compare across different assignment sets. Such a comparison must involve a full trade-off analysis through a social welfare function. Moreover, consumers may receive more or less subsidies under wealth-based subsidies compared to benefit-based subsidies.

Focusing on assignment sets rather than the allocation is natural for an indivisible good. Assignment sets appear to be important in practice. Many policies put more emphasis on one particular dimension of the environment, and relegate other dimensions. We implicitly argue that whether a consumer should consume a good is of primary importance, while the tax burden or actual subsidy amount for consumers is of secondary concern to policy makers.

We have taken as exogenous the possible information structures. We have ignored the possibility that consumers can manipulate information before it is made available to the regulator. A benefit-based policy may provide incentives for consumers to invest in effort to change their distributions of benefits. For example, if college scholarships are based on test scores, students may work harder to make their test results more favorable. For the short run, such incentives probably are unimportant, but they may be the dominant factor in the longer run.

Acknowledgements We thank seminar participants at Boston College, Boston University, Chinese University of Hong Kong, CSEF in Salerno, Duke University, Conference on the Economics of the Health Care and the Pharmaceutical Industry in Toulouse 2008, National Taiwan University, North American Summer Meetings of the Econometric Society 2007, Rutgers University, University of Bergen, University of Lausanne, Univesity of Liège, University of Michigan, and University of Oslo for their comments. We also thank Ingela Alger, Steve Coate, Ting Liu, Preston McAfee, Rich McLean, Dilip Mookherjee, Larry Samuelson, Wendelin Schnedler, and Thomas Sjostrom for their suggestions. Advice and comments from an editor and two referees are appreciated. The first author is grateful to the Fulbright Foundation of Italy for financial support.

\section{Appendix}

Proof of Proposition 1 Consider those $\ell \in[\underline{\ell}, \bar{\ell}] \cap\left[\underline{\ell}^{\prime}, \bar{\ell}^{\prime}\right]$. By construction, the policy $s(\ell)$ satisfies $U(w-s(\ell))+\ell=U(w)$. Any type $(w, \ell)$ consumer is indifferent between purchasing the good at price $t(w)$ and not if and only if he is indifferent at price $s(\ell)$. We show that $(w, \ell)$ belongs to $\alpha(t)$ if and only if it belongs to $\beta(s)$. 
Suppose $(w, \ell) \in \alpha(t)$ where $\alpha(t) \equiv\{(w, \ell): U(w-t(w))+\ell \geq U(w)\}$. We have

$$
U(w-t(w))+\ell \geq U(w-t(w))+\theta(w)=U(w)
$$

with a strict inequality if $\ell>\theta(w)$. Let $\hat{\ell}=\theta(w) \leq \ell$. By the definition of $s$, we have $U(w-s(\hat{\ell}))+\hat{\ell}=U(w)$. Because $\theta$ is strictly decreasing, for $\ell \geq \hat{\ell}$, there exists $\hat{w} \leq w$ such that

$$
U(\hat{w}-s(\ell))+\ell=U(\hat{w}) .
$$

But $w \geq \hat{w}$. Hence Eq. (30) implies

$$
U(w-s(\ell))+\ell \geq U(w)
$$

which says that $(w, \ell) \in \beta(s)$. We can use a symmetric argument to show that if $(w, \ell)$ does not belong to $\alpha(t)$, then it does not belong to $\beta(s)$.

Next consider $\ell \in\left[\underline{\ell}, \underline{\ell}^{\prime}\right]$ (if it exists). According to $\alpha(t)$, a type $(w, \ell)$ consumer does not purchase the good when $\ell<\underline{\ell}^{\prime}$, for all $w \in[\underline{w}, \bar{w}]$. By construction, $U\left(\bar{w}-s\left(\underline{\ell}^{\prime}\right)\right)+\underline{\ell}^{\prime}=U(\bar{w})$. Hence, $U\left(\bar{w}-s\left(\underline{\ell}^{\prime}\right)\right)+\ell<U(\bar{w})$, and therefore, $U\left(w-s\left(\underline{\ell}^{\prime}\right)\right)+\ell<U(w)$, all $w$. A type $(w, \ell)$ consumer does not purchase the good under $s(\ell)$ either.

Finally, consider $\ell \in\left[\bar{\ell}^{\prime}, \bar{\ell}\right]$ (if it exists). According to $\alpha(t)$, a type $(w, \ell)$ consumer purchases the good when $\ell>\bar{\ell}^{\prime}$, for all $w$. By construction, $U\left(\underline{w}-s\left(\bar{\ell}^{\prime}\right)\right)+\bar{\ell}^{\prime}=U(\underline{w})$. Hence $U\left(\underline{w}-s\left(\bar{\ell}^{\prime}\right)\right)+\ell>U(\underline{w})$, and therefore, $U\left(w-s\left(\bar{\ell}^{\prime}\right)\right)+\ell>U(w)$, all $w$. A type $(w, \ell)$ consumer always purchases the good under $s(\ell)$. This concludes the proof that $\alpha(t)=\beta(s)$.

Proof of Lemma 1 Consider a sequence $\left(w_{i}, \ell_{i}\right)$ that converges to $(w, \ell)$, and let $\left(w_{i}, \ell_{i}\right) \in \Omega$, each $i$. By assumption $U\left(w_{i}-t\left(w_{i}\right)\right)+\ell_{i} \geq U\left(w_{i}\right)$. Because $\ell_{i}$ converges to $\ell$, we have $U\left(w_{i}-t\left(w_{i}\right)\right)+\ell \geq U\left(w_{i}\right)$ and $\left(w_{i}, \ell\right) \in \Omega$, each $i$. Because $\Omega$ is also implementable by a benefit-based policy, we have $U\left(w_{i}-s(\ell)\right)+\ell \geq U\left(w_{i}\right)$. Now by assumption $w_{i}$ converges to $w$. So the continuity of $U$ implies that $(w, \ell) \in \Omega$. Since the graph of the boundary of a closed set is continuous, $s$ and $t$ must also be continuous.

Proof of Lemma 2 Let $\Omega$ be implementable simultaneously by $t(w)$ and $s(\ell)$. Let $(w, \ell)$ be a point on the indifference boundary so that $U(w-t(w))+\ell=U(w)$ and $U(w-s(\ell))+\ell=U(w)$. Consider a point $\left(w^{\prime}, \ell^{\prime}\right)$, where $w^{\prime}<w$ and $\ell^{\prime}<\ell$. Any $\left(w, \ell^{\prime}\right), \ell^{\prime}<\ell$, does not belong to $\Omega$ because $U(w-t(w))+\ell^{\prime}<U(w)$. Because $\Omega$ is implementable simultaneously by $t$ and $s$, we have $U\left(w-s\left(\ell^{\prime}\right)\right)+\ell^{\prime}<U(w)$, which implies $U\left(w^{\prime}-s\left(\ell^{\prime}\right)\right)+\ell^{\prime}<U\left(w^{\prime}\right)$ for $w^{\prime}<w$. Hence $\left(w^{\prime}, \ell^{\prime}\right) \notin \Omega$, and does not belong to the indifference boundary. A similar argument establishes that $\left(w^{\prime}, \ell^{\prime}\right)$ does not belong to the indifference boundary when $w^{\prime}>w$ and $\ell^{\prime}>\ell$.

We conclude that if $(w, \ell)$ and $\left(w^{\prime}, \ell^{\prime}\right)$ belong to the indifference boundary, either $w>w^{\prime}$ and $\ell<\ell^{\prime}$, or $w<w^{\prime}$ and $\ell>\ell^{\prime}$. 
Proof of Proposition 5 Let $s_{1}(\ell)$ and $s_{2}(\ell)$ satisfy Eq. (17). For each $\ell$ choose $\epsilon$ and $\delta$ such that

$$
U\left(\phi(\ell)-s_{2}(\ell)+\delta\right)+\ell=U\left(\phi(\ell)-s_{1}(\ell)+\epsilon\right) .
$$

By the continuity of $U$, such $\epsilon$ and $\delta$ exist. From (31), for each $\ell$ the values of $\epsilon$ and $\delta$ must follow:

$$
\frac{\mathrm{d} \epsilon}{\mathrm{d} \delta}=\frac{U^{\prime}\left(\phi(\ell)-s_{2}(\ell)+\delta(\ell)\right)}{U^{\prime}\left(\phi(\ell)-s_{1}(\ell)+\epsilon(\ell)\right)}>0 .
$$

As $\ell$ varies over its range, we obtain the functions $\epsilon(\ell)$ and $\delta(\ell)$. Now let the policy be $\left[s_{1}(\ell)+\epsilon(\ell), s_{2}(\ell)+\delta(\ell)\right]$, which also satisfies $(17)$. The collected revenue under $\left[s_{1}(\ell)+\epsilon(\ell), s_{2}(\ell)+\delta(\ell)\right]$ is

$$
\begin{aligned}
R\left(s_{1}(\ell)+\epsilon(\ell), s_{2}(\ell)+\delta(\ell)\right) \equiv & \int_{\beta\left(s_{1}, s_{2}\right)^{c}}\left[s_{1}(\ell)+\epsilon(\ell)\right] \mathrm{d} F(w) \mathrm{d} G(\ell) \\
& +\int_{\beta\left(s_{1}, s_{2}\right)}\left[s_{2}(\ell)+\delta(\ell)\right] \mathrm{d} F(w) \mathrm{d} G(\ell) \\
= & R\left(s_{1}, s_{2}\right)+\int_{\beta\left(s_{1}, s_{2}\right)^{c}} \epsilon(\ell) \mathrm{d} F(w) \mathrm{d} G(\ell) \\
& +\int_{\beta\left(s_{1}, s_{2}\right)} \delta(\ell) \mathrm{d} F(w) \mathrm{d} G(\ell) .
\end{aligned}
$$

For a given policy $\left[s_{1}(\ell), s_{2}(\ell)\right], R\left(s_{1}(\ell)+\epsilon(\ell), s_{2}(\ell)+\delta(\ell)\right)$ is monotone in $\epsilon$ due to (32). So there exist $\epsilon(\ell)$ and $\delta(\ell)$ such that $R\left(s_{1}(\ell)+\epsilon(\ell), s_{2}(\ell)+\delta(\ell)\right)=R\left(t_{1}, t_{2}\right)$.

Example 1 Let $U$ be the logarithmic function. Let a wealth-based policy be quasi-linear, $t(w)=a+b w$. The indifference boundary is given by $\ln (w-a-b w)+\ell=\ln w$, or $\ell=\theta(w) \equiv \ln \frac{w}{(w-a-b w)}$. We assume that the denominator is strictly positive; that is, $w-a-b w>0$. The derivative of $\theta$ is

$$
\frac{\mathrm{d} \theta}{\mathrm{d} w}=-\frac{a}{w(w-a-b w)} .
$$

The boundary is strictly decreasing if and only if $a>0$ (conditional on $w-a-b w>$ 0 ). The inverse of $\theta$ is $w=\phi(\ell) \equiv \frac{a e^{\ell}}{(1-b) e^{\ell}-1}$. Using in (10) to solve for $s$, we obtain the benefit-based policy

$$
s(\ell)=\frac{a\left(e^{\ell}-1\right)}{(1-b) e^{\ell}-1} .
$$


Example 2 Again let $U$ be the logarithmic function. Let a benefit-based policy be quasi-linear in $e^{-\ell}, s(\ell)=a+b e^{-\ell}$. The indifference boundary is given by $\ln (w-$ $\left.a-b e^{-\ell}\right)+\ell=\ln w$, or $w=\varphi(\ell) \equiv e^{\ell \frac{a+b e^{-\ell}}{e^{\ell}-1}}$. We assume that $w-a-b e^{-\ell}>0$. The derivative of $\varphi$ is

$$
\frac{\mathrm{d} \varphi}{\mathrm{d} \ell}=-\frac{(a+b) e^{-\ell}}{\left(e^{-\ell}-1\right)^{2}}
$$

The boundary is strictly decreasing if and only if $(a+b)>0$. The inverse of $\varphi$ is $\ell=\vartheta(w) \equiv \ln \frac{w+b}{(w-a)}$. By substituting $\ell$ in $U(w-t)+\ell=U(w)$ by $\vartheta(w)$ and solving for $t$, we obtain the wealth-based policy

$$
t(w)=\frac{w}{w+b}(a+b) \text {. }
$$

Example 3 Let $w$ and $\ell$ be uniformly and independently distributed over the interval $[0.5,1.5]$. Consider the wealth-based policy $t(w)=0.1+0.3 w$. We obtain the equivalent benefit-based policy $s(\ell)=\frac{0.1\left(e^{\ell}-1\right)}{0.7 e^{\ell}-1}$. From these, we compute the revenue that will be collected under each of these schemes:

$$
R(t) \equiv \int_{\alpha(t)} t(w) \mathrm{d} F(w) \mathrm{d} G(\ell)=0.393 \text { and } R(s) \equiv \int_{\beta(t)} s(\ell) \mathrm{d} F(w) \mathrm{d} G(\ell)=0.246 .
$$

In percentage terms we have:

$$
\frac{R(t)-R(s)}{R(s)}=60 \%
$$

\section{References}

Arrow K (1971) An utilitarian approach to the concept of equality in public expenditure. Q J Econ 85(3):409-415

Atkinson AB (1992) On targeting social security: the experience of western countries with family benefits. Paper presented at the World Bank Conference on Public Expenditure and the Poor: Incidence and Targeting, Washington DC

Besley T, Coate S (1991) Public provision of private goods and the redistribution of income. Am Econ Rev 81(4):979-984

Blackorby C, Donaldson D (1988) Cash versus kind, self-selection, and efficient transfers. Am Econ Rev 78(4):691-700

Blackorby C, Donaldson D (1994) Information and intergroup transfers. Am Econ Rev, Papers and Proceedings 84:440-447

Boadway R, Marchand M (1995) The use of public expenditures for redistributive purposes. Oxford Economic Papers, New Series 47(1):45-59

De Fraja G (2002) The design of optimal education policies. Rev Econ Stud 69:437-466

Grassi S, Ma CA (2009) Public sector rationing and private sector selection. Boston University Working 750 Paper

Mirrlees JA (1971) An exploration in the theory of optimum income taxation. Rev Econ Stud 38(114):175208

Patrinos HA, Ridao-Cano C, Sakellariou C (2006) Estimating the returns to education: accounting for heterogeneity in ability. The World Bank Policy Research Working Paper Series, 4040 